\title{
Reaction of $o$-Aminobenzamides with Dialkyl Carbonates and Ionic Liquids: A Novel one-pot, High-yield, Microwave-assisted Synthesis of 1-Alkylquinazoline-2,4-diones
}

\author{
Leticia Guerrero R. ${ }^{1}$ and Ignacio A. Rivero ${ }^{1,2 *}$ \\ ${ }^{1}$ Centro de Graduados e Investigación del Instituto Tecnológico de Tijuana. Apartado Postal 1166, Tijuana, B.C. 22000 \\ México.irivero@tectijuana.mx. \\ 2 Instituto Nacional de Investigaciones Nucleares, Departamento de Química. Carretera México Toluca S/N, La Marquesa, \\ Ocoyoacac, México, D.F. C.P. 52750.
}

Received December 6, 2011; accepted April 6, 2012

\begin{abstract}
A simple and efficient methodology for the synthesis of 1-alkyl-2,4-(1H,3H)-quinazolinediones from $o$-aminobenzamides has been developed. The novel one-pot cyclization-alkylation reactions are easier and faster than any other reported protocols and resulted in excellent yields (96-98\%) and high purities, making this methodology suitable for library synthesis in drug discovery efforts. By applying microwave irradiation at $130{ }^{\circ} \mathrm{C}$ and in the presence of ionic liquids, dimethyl or diethyl carbonates simultaneously act as carbonylating and alkylating agents.
\end{abstract}

Key words: Quinazolinedione, alkylation, carbonate, ionic liquid, microwave.

\section{Introduction}

Quinazoline-2,4-diones are an important class of heterocyclic compounds due to their wide array of pharmacological properties. They have been prepared over the years to serve as potential antihypertensive agents [1]. Pelanserine $4 \mathbf{h}\left(\mathrm{R}^{2}=\mathrm{H}\right)$ is one such potent molecule [2], structurally similar to the clinically used ketanserine [3]. Two natural alkaloids containing the quinazoline-2,4-dione ring skeleton, 1-methyl-3-(2'phenylethyl)-1H,3H-quinazoline-2,4-dione $\mathbf{4 g}\left(\mathrm{R}^{2}=\mathrm{Me}\right)$ and 1-methyl-3-[2'-(4'-methoxyphenyl)ethyl]-1 $H, 3 H$-quina-zoline2,4-dione $\mathbf{4 f}\left(\mathrm{R}^{2}=\mathrm{Me}\right)$ have been isolated from the seed husks of Zanthoxylum arborescens [4]. There are several synthetic pathways for their preparation [5], most of them starting from anthranilic acid derivatives.

Several years ago our research group reported a general methodology for $o$-aminobenzamides $(\mathrm{OAB})$ and quinazolinediones synthesis [5b] from primary amines with isatoic anhydride followed by the ring closure using triphosgene. In general, the preparation of 1-alkyl-2,4(1H,3H)-quinazolinediones 3 is achieved through a two steps sequence, i.e., the carbonylation of OAB 1 with ethyl chloroformate [5b], phosgene [2a] or triphosgene $[5 b]$ followed by the $\mathrm{N}$-alkylation of the resulting quinazolinediones 2 with an alkyl halide $\left(\mathrm{R}^{2} \mathrm{X}\right.$, Scheme 1) [6].

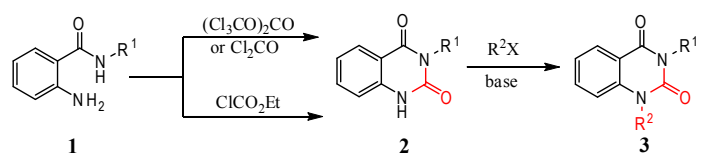

Scheme 1. Conventional synthesis of 1-alkyl-2,4(1H,3H)-quinazolinediones 3 .
Resumen: Se ha desarrollado una metodología eficiente para la sintesis de 1-alquil-2,4-(1H,3H)-quinazolinedionas a partir de $o$-aminobenzamidas. Es una reacción novedosa que realiza tanto la ciclación como la alquilación al mismo tiempo, es más fácil y rápida que cualquier otro protocolo, se obtienen con excelentes rendimientos (96-98\%) y alta pureza. Esta metodología es adecuada para la síntesis de bibliotecas que potencialmente promueven el desarrollo de nuevos fármacos. Mediante la irradiación por microondas a $130{ }^{\circ} \mathrm{C}$ y en presencia de líquidos iónicos, los carbonatos de dimetilo o de dietilo pueden actuar como agentes para la carbonilación y la alquilación.

Palabras clave: Quinazolinediona, alquilación, carbonato, liquido iónico, microondas.

These procedures are not eco-friendly due to risks associated with toxic and corrosive reagents such as phosgene, carcinogenic alkyl halides and lachrymatory ethyl chloroformate. Likewise, use of ethyl chloroformate led to a complex mixture of products. The desired products of high purity are obtained upon a tedious and difficult work-up.

A safer, easy and eco-friendly alternative can be conceived by using low-toxic dialkyl carbonates. Dialkyl carbonates simultaneously act as alkoxycarbonylating and alkylating agents in only few instances. Among them, there is a case of oximes with an $\alpha-\mathrm{CH}_{2}$ group for the synthesis of 3-methyl-4,5-disubstituted-4-oxazolin-2-ones [7]. More recently, the preparation of $\mathrm{N}$-methylbenzoxazol-2-ones from $o$-aminophenol and dimethyl carbonate with catalytic $\mathrm{Pb}(\mathrm{OAc})_{2}$ [8] or from $o$-aminophenol and dialkyl carbonates with catalytic $\mathrm{K}_{2} \mathrm{CO}_{3}$ was reported [9]. Our research group discovered that DMC and DEC can also function effectively for the O-alkylation of carboxylic acids and phenols under hard conditions, 1,2-dimethylimidazole (DMI) is employed as the catalyst [10].

In recent years, significant progress has been made in the application of ionic liquid as a new kind of green solvent and catalysts organic with unique chemical and physical properties, have attracted growing interest, and many catalytic reactions proceeded in ionic liquids with excellent performance [11]. Very recently the utilization of ionic liquid for the cycloaddition of $\mathrm{CO}_{2}$ to epoxides to produce cyclic carbonates has been extensively studied [12]. On the other hand, microwave has been employed for the promotion of a variety of organic transformations leading to faster and cleaner reactions when compared to conventional heating [13]. Since use of both ionic liquid and microwaves for organic transformations is still not a 
widely applied strategy, we decided to probe whether there is any advantage for this approach.

To our knowledge, no study has so far been made to examine the use of ionic liquid as catalyst for the synthesis of 1-alkylated 2,4-quinazolinediones from $\mathrm{OAB}$ and dialkyl carbonates. We report herein an efficient and novel synthesis of 1-alkyl-2,4-quinazolinediones $\mathbf{4}$ where dialkyl carbonates act at the same time as carbonylating and alkylating agents in the presence of $[\mathrm{bmim}][\mathrm{Cl}]$ or $\left[\mathrm{Bu}_{4} \mathrm{~N}\right][\mathrm{Cl}]$ ionic liquids (Scheme 2). This protocol exploits an unusual simultaneous cyclization-alkylation using ionic liquids catalyst and microwave irradiation. Since total conversions were achieved in all the cases, clean products can be isolated after simple aqueous work-up.

\section{Results and Discussion}

Initial experiments were carried out using diethyl carbonate (DEC) and dimethyl carbonate (DMC). A mixture of 2-amino$\mathrm{N}$-benzylbenzamide (1c) $(0.1 \mathrm{~g}, 0.44 \mathrm{mmol}), \mathrm{DEC}$ or DMC (1 $\mathrm{mL}$ ) and $\mathrm{K}_{2} \mathrm{CO}_{3}$ (3 equiv) was made to react at temperatures of $120^{\circ} \mathrm{C}, 140{ }^{\circ} \mathrm{C}, 160^{\circ} \mathrm{C}$ and for different time intervals, no product could be detected. The same experiments were also carried out in the presence of $N, N$-dimethylformamide (DMF, $1 \mathrm{~mL}$ ) as co-solvent. Under these conditions at $160^{\circ} \mathrm{C}$ after 16 min only traces of product were observed.

Ionic liquids have been shown to promote a variety of organic transformations effectively. Based on these precedents, we were particularly interested in examining the advantages of employing stoichiometric amounts of ionic liquid for the reactions, since most of the ionic liquids are expensive when used as solvents. This study was first conducted by treating $1 \mathbf{c}$ with DEC and DMF in the presence of a variety of ionic liquids under certain conditions (Table 1). The ionic liquid ([bmim] $[\mathrm{Cl}]$ ) was selected to determine optimal reaction conditions of monitoring by HPLC until a trace or no starting material was de-

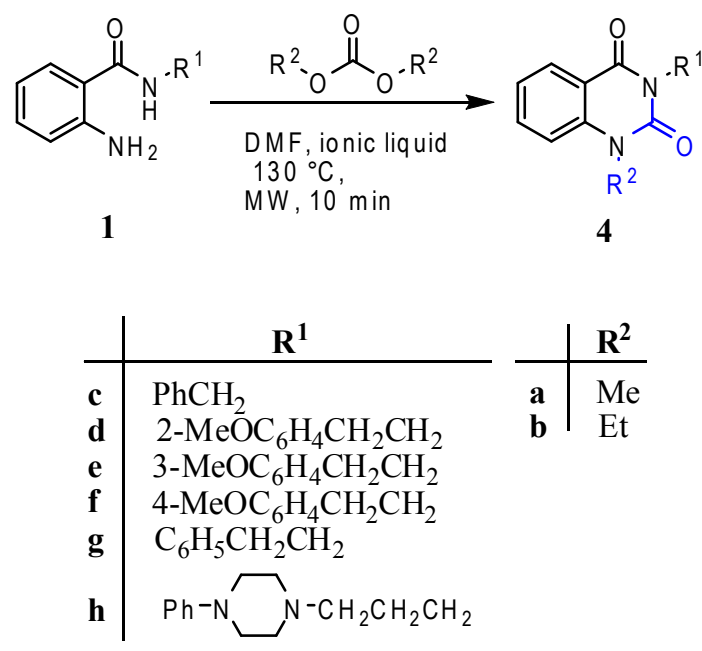

Scheme 2. Reaction of $N$-alkyl-2-aminobenzamides (1) with dialkyl carbonates. tected, finding it in $130{ }^{\circ} \mathrm{C}$ and 10 minutes. No by-products were formed. Using these conditions the ionic liquids selected were screened for the same reaction (Table 1).

In reactions with DEC total conversions were observed when 1 equiv. of imidazolium-type ionic liquid was used except when the counter ion was $\mathrm{PF}_{6}^{-}$, and the reaction did not proceeded without ionic liquid. The presence of ionic liquids in the reaction contributes to a better absorption of microwave radiation by more efficient heat distribution.

In comparison, tetrabutylammonium chloride (TBAC) (Table 1, entry 2) was found to be less effective, yields were low although the reaction temperature was increased up to $160{ }^{\circ} \mathrm{C}$, and undesired by-products were formed. The same procedure was applied to the reactions with DMC and the results summarized in Table 1 show the opposite to those observed with DEC. Imidazolium-type ionic liquids were found to be less effective, even when temperatures and equivalents are increased. However, employing TBAC with DMC, total conversion in $10 \mathrm{~min}$ was observed. The results demonstrate that the type of cations and anions of the ionic liquids have a strong impact on the total conversion and selectivity. Ionic liquids that contain a chloride anion showed excellent catalytic activity, while imidazolium cations were proven to be the best in the synthesis using DEC, the non-imidazolium IL was the best in the DMC series.

The effect of the ionic liquid amount was then investigated, selecting $[\mathrm{bmim}][\mathrm{Cl}]$ for $\mathrm{DEC}$ reactions due to their low cost and less quantity of wastes in products. A set of reactions were performed using a solution of 1c $(0.1 \mathrm{~g}, 0.44 \mathrm{mmol})$ in DEC or DMC $(1 \mathrm{~mL})$ and DMF $(1 \mathrm{~mL})$ to which different amounts of $[\mathrm{bmim}][\mathrm{Cl}]$ or $\left[\mathrm{Bu}_{4} \mathrm{~N}\right][\mathrm{Cl}]$ in the range of $0.2-1.0$ molar equivalents with respect to the substrate were added. Experiments were run for $10 \mathrm{~min}$ at $130{ }^{\circ} \mathrm{C}$. Results are reported in Figure 1.

Data clearly show that the IL can be used as an additive in the case of DMC and less than 0.75 equivalents for DEC reactions. The reaction rates and yields depend greatly on the structure of

Table 1. Effect of ionic liquids on the synthesis of 1-alkyl-3-benzyl$1 H, 3 H$-quinazoline-2,4-diones (4cb and $\mathbf{4 c a}$ ).

\begin{tabular}{ccccc}
\hline & \multicolumn{2}{c}{ DEC } & \multicolumn{2}{c}{ DMC } \\
\hline Entry & Ionic Liquid $^{\mathrm{a}}$ & $\begin{array}{c}{[\%]} \\
(\mathbf{4 c b})^{\mathrm{b}}\end{array}$ & Ionic Liquid $^{\mathrm{a}}$ & $\begin{array}{c}{[\%]} \\
(\mathbf{4 c a})^{\mathrm{b}}\end{array}$ \\
\hline 1 & none & 0 & none & 0 \\
2 & $\mathrm{Bu}_{4} \mathrm{~N} \mathrm{Cl}$ & 43 & $\mathrm{Bu}_{4} \mathrm{~N} \mathrm{Cl}$ & 98 \\
3 & {$\left[\mathrm{bmim}^{\mathrm{N}} \mathrm{PF}_{6}\right.$} & 0 & {$[\mathrm{bmim}] \mathrm{PF}_{6}$} & 0 \\
4 & {$[\mathrm{emim}] \mathrm{Cl}$} & $>98$ & {$[\mathrm{emim}] \mathrm{Cl}$} & 42 \\
5 & {$[\mathrm{hmim}] \mathrm{Cl}$} & $>98$ & {$[\mathrm{hmim}] \mathrm{Cl}$} & 43 \\
6 & {$[\mathrm{bmim}] \mathrm{Cl}$} & 96 & {$[\mathrm{bmim}] \mathrm{Cl}$} & 40 \\
\hline
\end{tabular}

a $[$ bmim $]=1$-butyl-3-methylimidazolium; $[$ emim $]=1$-ethyl-3-methylimidazolium; $[\mathrm{hmim}]=1$-hexyl-3-methylimidazolium.

b Yields were determined by HPLC analysis of the reaction mixture at the end of the reaction time indicated $(10 \mathrm{~min})$. (carrier solvent: methanol/water (80:20), $14.5 \mathrm{~min}$ ). 


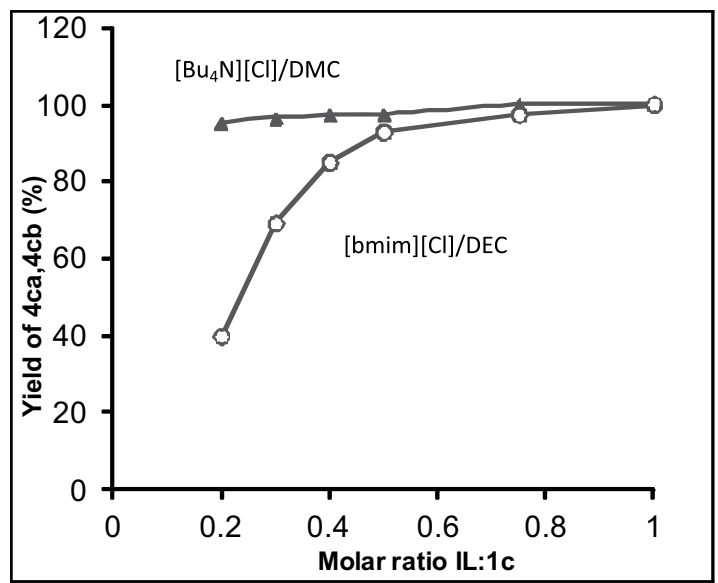

Fig. 1. Isolated yield of $\mathbf{4 c a}$ and $\mathbf{4 c b}$ in the reaction of OAB 1c, DEC or DMC with different amounts of ionic liquid selected.

the cation as well as the nucleophilicity of the anion. However, the structure of the solute entities in IL is still unclear. Interconversion of coordinatively unsaturated-saturated species is a key feature in catalyst function, fundamental studies on the coordination behavior of ionic liquids with different dialkyl carbonates are required in order to understand this area more clearly.

The uncommon reactions are believed to proceed through a sequence of $\mathrm{B}_{\mathrm{AC}} 2$ (Base-catalyzed, bimolecular attack on the acyl group) $/ B_{\mathrm{AL}} 2 \mathrm{~N}$ (Base-promoted, bimolecular attack on the alkyl group) sequence $[9,14]$. Ionic liquid performs as a catalyst to yield to the 1-alkylated 2,4-(1H,3H)-quinazolinediones. A mechanistic study to elucidate the exact role of ionic liquids is required.

To investigate the synthetic utility of this reaction, a variety of $o$-aminobenzamides were carbonylated-alkylated using $\mathrm{DMC}$ and DEC with 0.7 equiv of [bmim] [Cl] and 0.3 equiv of $\left[\mathrm{Bu}_{4} \mathrm{~N}\right][\mathrm{Cl}]$ respectively and DMF as co-solvent (Table 2).

Table 2. Synthesis of quinazoline-2,4(1H,3H)-diones 4 with dialkyl carbonates and ionic liquid $\left(\mathrm{MW}, 130^{\circ} \mathrm{C}, 10 \mathrm{~min}\right)$. [DMC $\left(\left[\mathrm{Bu}_{4} \mathrm{~N}\right][\mathrm{Cl}]\right)$ and DEC ([bmim $][\mathrm{Cl}])]$.

\begin{tabular}{|c|c|c|c|}
\hline Product & $\mathrm{R}^{1}$ & $\mathrm{R}^{2}$ & $4[\%]^{a, b}$ \\
\hline $4 \mathrm{ca}$ & \multirow[t]{2}{*}{$\mathrm{PhCH}_{2}$} & $\mathrm{Me}$ & 96 \\
\hline $4 \mathrm{cb}$ & & Et & 98 \\
\hline $4 d a$ & \multirow{2}{*}{ 2- $\mathrm{MeOC}_{6} \mathrm{H}_{4} \mathrm{CH}_{2} \mathrm{CH}_{2}$} & $\mathrm{Me}$ & 97 \\
\hline $4 d b$ & & $\mathrm{Et}$ & 96 \\
\hline 4ea & \multirow[t]{2}{*}{$3-\mathrm{MeOC}_{6} \mathrm{H}_{4} \mathrm{CH}_{2} \mathrm{CH}_{2}$} & $\mathrm{Me}$ & 97 \\
\hline $4 \mathrm{eb}$ & & Et & 97 \\
\hline $4 \mathrm{fa}$ & \multirow[t]{2}{*}{$4-\mathrm{MeOC}_{6} \mathrm{H}_{4} \mathrm{CH}_{2} \mathrm{CH}_{2}$} & $\mathrm{Me}$ & 98 \\
\hline $4 \mathrm{fb}$ & & Et & 98 \\
\hline 4ga & \multirow[t]{2}{*}{$\mathrm{PhCH}_{2} \mathrm{CH}_{2}$} & $\mathrm{Me}$ & 98 \\
\hline $4 g b$ & & Et & 97 \\
\hline \multirow{2}{*}{$\begin{array}{l}\text { 4ha } \\
4 \mathrm{hb}\end{array}$} & \multirow{2}{*}{$\mathrm{Ph}-\mathrm{N} \longrightarrow \mathrm{N}-\mathrm{CH}_{2} \mathrm{CH}_{2} \mathrm{CH}_{2}$} & $\mathrm{Me}$ & 97 \\
\hline & & $\mathrm{Et}$ & 96 \\
\hline
\end{tabular}

${ }^{a}$ Identity of products was confirmed by ${ }^{1} \mathrm{H}$ and ${ }^{13} \mathrm{C}$ NMR. ${ }^{\mathrm{b}}$ Isolated yield based on starting material 1 .
All the reactions were complete in $10 \mathrm{~min}$. Since conversions were achieved in a $100 \%$ in all cases, clean products can be isolated by addition of water to 'precipitate' the products due to their complete insolubility in water. Light yellow solids that formed were separated by filtration and made carbonate free by washing with water. After drying, the product has the correct melting point without recrystallization. Due to its purity some of the products can be crystallized to give single crystals suitable for X-ray diffraction studies, e.g., $\mathbf{4 f b}$ (Figure 2) [15].

In all cases, the reaction proceeds with very high yields in isolated products. Ionic liquids and dialkyl carbonates efficiently perform the simultaneous carbonylating and alkylating of $o$-aminobenzamides under very simple conditions. COSY spectra support the identity of product $\mathbf{4 h b}$ Table 2 entry $6 R_{2}$ $=$ Et (supporting information) [15].

\section{Conclusions}

We have developed and optimized an unusual, efficient and clean cyclization-alkylation protocol for the formation of 2,4$(1 H, 3 H)$-quinazolinediones from $\mathrm{OAB}$ and dialkyl carbonates using ionic liquids and microwave irradiation. The notable advantages of this method are (a) fast and simple work-up of products purification, (b) low quantities of ionic liquid with very high yields and purity, (c) potential separation and recycling of catalysts and solvents (d) valuable environmental and synthetic advantages are the use of non toxic compounds and catalyst, and the fact that neither organic nor toxic inorganic byproducts are formed. Further investigations into the mechanism are required to elucidate the exact role of ionic liquids leading to the 1-alkylated 2,4-(1H,3H)-quinazolinediones product.

\section{Experimental}

General. All reagents and solvents were purchased from commercial suppliers and used without further purification. ${ }^{1} \mathrm{H}$ and ${ }^{13} \mathrm{C}$ NMR spectra were recorded at $200 \mathrm{MHz}$ and $50.289 \mathrm{MHz}$, respectively, on a Varian Mercury $200 \mathrm{MHz}$ spectrometer in $\mathrm{CDCl}_{3}$ with TMS as internal standard. Mass spectra were obtained on an 1100 series LC/MSD Trap, Agilent SL spectrometer by electrospray insertion. Melting points were measured on

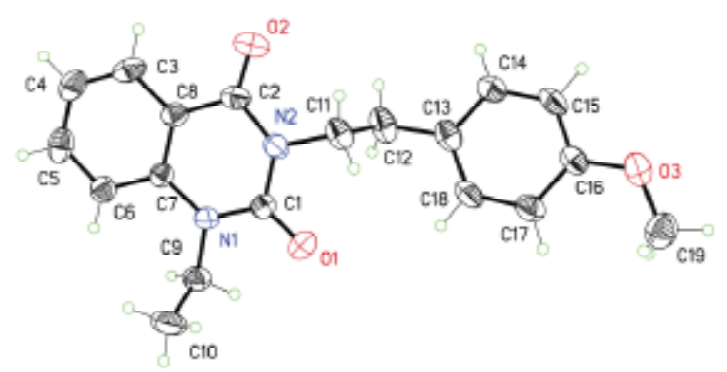

Fig. 2. X-Ray structure of 1-Ethyl-3-[2'-(4'-methoxyphenyl)ethyl]$1 H, 3 H$-quinazoline-2,4-dione $(4 \mathrm{fb}) .{ }^{15}$ 
an electrothermal 88629 apparatus. Infrared (IR) spectra were recorded on a Perkin Elmer FT-IR 1600 spectrometer. The MW experiments were performed in a self-tuning single mode CEM Discover ${ }^{\mathrm{TM}}$ Focused Synthesizer apparatus; the instrument continuously adjusted the applied wattage to maintain the desired temperature. The $\mathrm{OAB} 1$ were prepared according to the general methodology reported $[6 \mathrm{c}]$.

1-Methyl 2,4-(1H,3H)-quinazolinediones. General procedure. A $10 \mathrm{~mL}$ pressure-rated vial was charged with $\mathrm{OAB}$ $(1,0.10 \mathrm{~g})$, ionic liquid $\left[\mathrm{Bu}_{4} \mathrm{~N}\right][\mathrm{Cl}](0.3$ equiv), DMF $(1 \mathrm{~mL})$ and DMC $(1 \mathrm{~mL}, 8.8 \mathrm{mmol})$. The vial was sealed and the mixture under stirring was irradiated at $130{ }^{\circ} \mathrm{C}$ for $10 \mathrm{~min}$ in the microwave reactor ( $200 \mathrm{~W}$ power level). After letting the reaction mixture cool, water $(3 \mathrm{~mL})$ was added and this mixture was set aside. Light yellow solid that formed was separated by filtration and made carbonate free by washing with water. The solid product not need further purification and must be dried to confirm by NMR spectroscopy.

1-Methyl-3-benzyl-1H,3H-quinazoline-2,4-dione $(4 \mathrm{c}, \mathrm{a})$. 1c $(0.10 \mathrm{~g}, 0.44 \mathrm{mmol})$ gave a white solid $(114.8 \mathrm{mg}, 98 \%) ; \mathrm{mp}$ 103-106 ${ }^{\circ} \mathrm{C}$ (lit. [6c] mp 103-106 ${ }^{\circ} \mathrm{C}$ ). IR (KBr): 3032, 2918, $1700,1652,1604,1480 \mathrm{~cm}^{-1} .{ }^{1} \mathrm{H}$ NMR $\left(200 \mathrm{MHz}, \mathrm{CDCl}_{3}\right): \delta$ $8.22(1 \mathrm{H}, \mathrm{dd}, J=1.6,7.8 \mathrm{~Hz}, \mathrm{Ar}-\mathrm{H}), 7.64(1 \mathrm{H}, \mathrm{ddd}, J=1.6$, 7.3, 8.5 Hz, Ar-H), 7.52 (2H, dd, $J=1.7,7.8 \mathrm{~Hz}, \mathrm{Ar}-\mathrm{H}), 7.34-$ $7.13(5 \mathrm{H}, \mathrm{m}, \mathrm{Ar}-\mathrm{H}), 5.27\left(2 \mathrm{H}, \mathrm{s}, \mathrm{CH}_{2}\right), 3.57\left(3 \mathrm{H}, \mathrm{s}, \mathrm{CH}_{3}\right) .{ }^{13} \mathrm{C}$ NMR (50 MHz, $\left.\mathrm{CDCl}_{3}\right): \delta 161.7,150.9,140.9,137.0,135.1$, $129.7,129.0,128.3,127.5,122.9,115.5,113.5,44.9,30.7$. ESI-MS: $m / z 288.9[\mathrm{M}+\mathrm{Na}]^{+}$.

1-Methyl-3-[2'-(2'-methoxyphenyl)ethyl]-1H,3H-quinazoline-2,4-dione (4d,a). 1d $(0.10 \mathrm{~g}, 0.37 \mathrm{mmol})$ gave a white solid 4d,a (111.4 mg, 97\%); mp. 158-163 ${ }^{\circ} \mathrm{C}$. (Lit. [6c] mp. 155-163 ${ }^{\circ} \mathrm{C}$ ); IR (KBr): 3035, 2943, 2848, 1703, 1651, 1608, $1484,1243 . \mathrm{cm}^{-1}$; ${ }^{1} \mathrm{H}$ NMR $\left(200 \mathrm{MHz}, \mathrm{CDCl}_{3}\right): \delta 8.21(1 \mathrm{H}$, dd, $J=1.6,7.8 \mathrm{~Hz}, \mathrm{Ar}-\mathrm{H}), 7.65(1 \mathrm{H}$, ddd, $J=1.7,7.4,8.5$ $\mathrm{Hz}, \mathrm{Ar}-\mathrm{H}), 7.30-6.85(6 \mathrm{H}, \mathrm{m}, \mathrm{Ar}-\mathrm{H}), 4.28(2 \mathrm{H}$, ddd, $J=5.2$, 7.8, 7.8 Hz, N-CH $)_{2}, 3.80\left(3 \mathrm{H}, \mathrm{s}, \mathrm{O}-\mathrm{CH}_{3}\right), 3.60\left(3 \mathrm{H}, \mathrm{s}, \mathrm{N}-\mathrm{CH}_{3}\right)$, $2.95\left(2 \mathrm{H}, \mathrm{ddd}, J=5.2,7.8,7.8 \mathrm{~Hz}, \mathrm{Ar}_{-} \mathrm{CH}_{2}\right) \mathrm{ppm} ;{ }^{13} \mathrm{C} \mathrm{NMR}$ $\left(50 \mathrm{MHz}, \mathrm{CDCl}_{3}\right): \delta 157.6,134.9,130.6,128.9,127.7,122.8$, $120.41,113.4,110.2,55.3,41.6,30.6,26.7 \mathrm{ppm}$. MS m/z: $310.9[\mathrm{M}+\mathrm{H}]^{+}$.

1-Methyl-3-[2'-(3'-methoxyphenyl)ethyl]-1H,3H-quinazoline-2,4-dione $(4 \mathrm{e}, \mathrm{a})$. $1 \mathrm{e}(0.10 \mathrm{~g}, 0.37 \mathrm{mmol})$ gave a white solid 4e,a (111.5 mg, 97\%); mp. 131-133 ${ }^{\circ} \mathrm{C}$. (Lit. [6c] mp. 131-133 ${ }^{\circ} \mathrm{C}$ ); IR (KBr): 3025, 2945, 2833, 1799, 1656, 1604, $1483,1258 \mathrm{~cm}^{-1}$; ${ }^{1} \mathrm{H}$ NMR $\left(200 \mathrm{MHz}, \mathrm{CDCl}_{3}\right): \delta 8.23(1 \mathrm{H}, \mathrm{dd}$, $J=1.6,7.9 \mathrm{~Hz}, \mathrm{Ar}-\mathrm{H}), 7.63(1 \mathrm{H}, \mathrm{ddd}, J=1.6,7.2,8.4 \mathrm{~Hz}$, Ar-H), 7.25-7.15 (6H, m, Ar-H), 6.78 (1H, ddd, $J=1.0,2.5$, $8.2 \mathrm{~Hz}, \mathrm{Ar}-\mathrm{H}), 4.30$ (2H, ddd, $J=5.4,7.6,7.6 \mathrm{~Hz}, \mathrm{~N}-\mathrm{CH}_{2}$ ), $3.80\left(3 \mathrm{H}, \mathrm{s}, \mathrm{O}-\mathrm{CH}_{3}\right), 3.55\left(3 \mathrm{H}, \mathrm{s}, \mathrm{N}-\mathrm{CH}_{3}\right), 2.98(2 \mathrm{H}, \mathrm{ddd}, J=$ 5.1, 7.6, 7.6 Hz, Ar- $\left.\mathrm{CH}_{2}\right)$ ppm; ${ }^{13} \mathrm{C}$ NMR $\left(50 \mathrm{MHz}, \mathrm{CDCl}_{3}\right): \delta$ $162.5,159.9,148.5,140.5,132.8,132.4,129.7,127.0,126.4$, $122.7,117.4,116.8,116.3,112.1,55.1,40.7,335.7$ ppm. ESIMS $(m / z): 332.9[\mathrm{M}+\mathrm{Na}]^{+}$.

1-Methyl-3-[2'-(4'-methoxyphenyl)ethyl]-1 $\mathrm{H}, 3 \mathrm{H}$-quinazoline-2,4-dione (4f,a). 1f $(0.10 \mathrm{~g}, 0.37 \mathrm{mmol})$ gave a white solid 4f,a (112.5 mg, 98\%); mp. 134-136 ${ }^{\circ} \mathrm{C}$. (Lit. [16] mp. 133-134 ${ }^{\circ} \mathrm{C}$ ); IR (KBr): $3025,2928,1700,1647,1600,1400$, 1261. $\mathrm{cm}^{-1} ;{ }^{1} \mathrm{H}$ NMR $\left(200 \mathrm{MHz}, \mathrm{CDCl}_{3}\right): \delta 8.21(1 \mathrm{H}, \mathrm{dd}, J=$ $1.6,7.8 \mathrm{~Hz}, \mathrm{Ar}-\mathrm{H}), 7.69$ (1H, ddd, $J=1.7,7.4,8.5 \mathrm{~Hz}, \mathrm{Ar}-\mathrm{H})$, 7.30-7.18 (6H, m, Ar-H), $6.80(1 \mathrm{H}, \mathrm{ddd}, J=1.0,2.5,8.2 \mathrm{~Hz}$, Ar-H), $4.26\left(2 \mathrm{H}\right.$, ddd, $\left.J=5.2,7.8,7.8 \mathrm{~Hz}, \mathrm{~N}-\mathrm{CH}_{2}\right), 3.80(3 \mathrm{H}$, $\left.\mathrm{s}, \mathrm{O}-\mathrm{CH}_{3}\right), 3.59\left(3 \mathrm{H}, \mathrm{s}, \mathrm{N}-\mathrm{CH}_{3}\right), 2.95(2 \mathrm{H}, \mathrm{ddd}, J=5.2,7.8$, $\left.7.8 \mathrm{~Hz}, \mathrm{Ar}-\mathrm{CH}_{2}\right) \mathrm{ppm} ;{ }^{13} \mathrm{C} \mathrm{NMR}\left(50 \mathrm{MHz}, \mathrm{CDCl}_{3}\right): \delta 161.8$, 158. 2, 140.5, 130.1, 129.2, 124.2, 114.0, 113.6, 55.5, 43.7, 33.4, $31.0 \mathrm{ppm}$. ESI-MS $(\mathrm{m} / \mathrm{z}): 311.2[\mathrm{M}+\mathrm{H}]^{+}$.

1-Methyl-3-(2'-phenylethyl)-1 $\mathrm{H}, 3 \mathrm{H}$-quinazoline-2,4-dione (4g,a). $1 \mathrm{~g}(0.10 \mathrm{~g}, 0.41 \mathrm{mmol})$ gave a white solid $\mathbf{4 g}$,a (112.6 mg, 98\%); mp 99-101 ${ }^{\circ} \mathrm{C}$ (Lit. [16] mp. 100-102 ${ }^{\circ} \mathrm{C}$ ); IR (KBr): $3042,2929,1701,1654,1610,1481 \mathrm{~cm}^{-1},{ }^{1} \mathrm{H}$ NMR $(200 \mathrm{MHz}$, $\left.\mathrm{CDCl}_{3}\right): \delta 8.23(1 \mathrm{H}, \mathrm{dd}, J=1.7,7.8 \mathrm{~Hz}, \mathrm{Ar}-\mathrm{H}), 7.65(1 \mathrm{H}, \mathrm{ddd}$, $J=1.7,7.3,8.4 \mathrm{~Hz}$, Ar-H), 7.34-7.16 (7H, m, Ar-H), 4.29 $\left(2 \mathrm{H}\right.$, ddd, $\left.J=5.6,7.8,7.8 \mathrm{~Hz}, \mathrm{~N}-\mathrm{CH}_{2}\right), 3.57\left(3 \mathrm{H}, \mathrm{s}, \mathrm{N}-\mathrm{CH}_{3}\right)$, $2.96\left(2 \mathrm{H}, \mathrm{ddd}, J=5.2,8.0,8.0 \mathrm{~Hz}, \mathrm{Ar}_{-} \mathrm{CH}_{2}\right) \mathrm{ppm} ;{ }^{13} \mathrm{C} \mathrm{NMR}$ $\left(50 \mathrm{MHz}, \mathrm{CDCl}_{3}\right): \delta 161.3,150.5,140.2,138.3,134.8,129.9$, 128.7, 128.6, 128.2, 126.2, 122.7,120.9, 113.25, 43.3, 33.9, $30.6 \mathrm{ppm}$. ESI-MS $(\mathrm{m} / \mathrm{z}): 281.1[\mathrm{M}+\mathrm{H}]^{+}$.

1-Methyl-3-[3'-(4'-phenylpiperazin-1-yl)propyl]-1 H,3Hquinazoline-2,4-dione $(\mathbf{4 h}, \mathbf{a})$. $1 \mathrm{~h}(0.10 \mathrm{~g}, 0.29 \mathrm{mmol})$ gave a white solid 4h,a (106.46 mg, 97\%); IR (KBr): 3010, 2937, 2880, 1695, 1647, 1604, 1223, $1110 \mathrm{~cm}^{-1}$. ${ }^{1} \mathrm{H}$ NMR $(200 \mathrm{MHz}$, $\left.\mathrm{CDCl}_{3}\right): \delta 8.18(1 \mathrm{H}, \mathrm{dd}, J=1.6,7.9 \mathrm{~Hz}, \mathrm{Ar}-\mathrm{H}), 7.62(1 \mathrm{H}, \mathrm{ddd}$, $J=1.6,7.4,8.4 \mathrm{~Hz}, \mathrm{Ar}-\mathrm{H}), 7.45(1 \mathrm{H}, \mathrm{d}, J=8.4 \mathrm{~Hz}, \mathrm{Ar}-\mathrm{H})$, 7.25-7.11 (3H, m, Ar-H), 6.88-6.75 (3H, m, Ar-H), 4.22-4.04 $\left(2 \mathrm{H}, \mathrm{m}, \mathrm{CON}-\mathrm{CH}_{2}\right), 3.58\left(3 \mathrm{H}, \mathrm{s}, \mathrm{N}-\mathrm{CH}_{3}\right), 3.18(4 \mathrm{H}, \mathrm{m}, \mathrm{N}-\mathrm{CH}-$ ${ }_{2}$ axial) $2.65-2.40\left(6 \mathrm{H}, \mathrm{m}, \mathrm{N}-\mathrm{CH}_{2}\right), 1.86\left(2 \mathrm{H}, \mathrm{m}, \mathrm{CH}_{2}\right) \mathrm{ppm} ;{ }^{13} \mathrm{C}$ NMR (50 MHz, $\left.\mathrm{CDCl}_{3}\right): \delta 161.8,151.3,148.8,140.5,135.0$, $129.1,128.9,128.8,122.8,119.5,115.9,112.8,56.1,53.0$, 49.1, 40.5, 30.6, 24.8 ppm. MS m/z:391.2 [M+Na] $]^{+}$.

1-Ethyl 2,4-(1H,3H)-quinazolinediones. General procedure. A designed $10 \mathrm{~mL}$ pressure-rated vial was charged with substrate $\mathrm{OAB}(1,0.10 \mathrm{~g})$, ionic liquid $[\mathrm{bmim}][\mathrm{Cl}](0.70$ equiv), DMF $(1 \mathrm{~mL})$ and DEC $(1 \mathrm{~mL}, 6.5 \mathrm{mmol})$. The vial was sealed and the mixture under stirring was irradiated at $130^{\circ} \mathrm{C}$ for 10 minutes in the microwave reactor $(200 \mathrm{~W}$ power level). After letting the reaction mixture cool, water $(3 \mathrm{~mL})$ was added and this mixture was set aside. Light yellow solid that formed was separated by filtration and made carbonate free by washing with water. The solid product not need further purification and must be dried to confirm by NMR spectroscopy.

1-Ethyl-3-benzyl-1 $H, 3 H$-quinazoline-2,4-dione $(4 \mathrm{c}, b)$. 1c $(0.10 \mathrm{~g}, 0.39 \mathrm{mmol})$ gave a white solid $\mathbf{4 c}, \mathbf{b}(107.1 \mathrm{mg}, 98 \%)$; mp. 103-105 ${ }^{\circ} \mathrm{C}$. (Lit. [16] mp. 133-134 ${ }^{\circ} \mathrm{C}$ ); IR (KBr): 3028 , 2974, 2922, 1701, 1657, 1605, $1483 \mathrm{~cm}^{-1}$; ${ }^{1} \mathrm{H}$ NMR $(200 \mathrm{MHz}$, $\mathrm{CDCl}_{3}$ ): $\delta 8.21$ (dd, $\left.1 \mathrm{H}, J=1.6,7.8 \mathrm{~Hz}, \mathrm{Ar}-\mathrm{H}\right), 7.69$ (ddd, $1 \mathrm{H}$, $J=1.7,7.4,8.5 \mathrm{~Hz}, \mathrm{Ar}-\mathrm{H}), 7.30-7.18(\mathrm{~m}, 6 \mathrm{H}, \mathrm{Ar}-\mathrm{H}), 7.52-$ $7.12(\mathrm{~m}, 8 \mathrm{H}, \mathrm{Ar}-\mathrm{H}), 5.27$ (s, 2H, N-CH $\left.{ }_{2}\right), 4.16$ (q, $2 \mathrm{H}, J=7$ $\mathrm{Hz} \mathrm{CH} \mathrm{CH}_{2}$ ), 1.32 (t, $\left.3 \mathrm{H}, J=7.0 \mathrm{~Hz}, \mathrm{CH}_{3}\right), \mathrm{ppm} ;{ }^{13} \mathrm{C} \mathrm{NMR}$ $\left(50 \mathrm{MHz}, \mathrm{CDCl}_{3}\right): \delta 163.8,150.8,139.5,137.0,135.4,129.3$, 127.6, 122.7, 115.8, 113.3, 44.8, 38.8, 12.5 ppm. ESI-MS $(\mathrm{m} /$ z):281.1 $[\mathrm{M}+\mathrm{H}]^{+}$. 
1-Ethyl-3-[2'-(2'-methoxyphenyl)ethyl]-1H,3H-quinazoline-2,4-dione (4d,b). 1d (0.10 g, $0.37 \mathrm{mmol})$ gave a white solid 4d,b (115.2 mg, 96\%); mp 128-130 ${ }^{\circ} \mathrm{C}$; (Lit. [16] mp. 128-130 ${ }^{\circ} \mathrm{C}$ ); IR (KBr): 3029, 2915, 2848, 1701, 1657, 1605, $1483,1240 \mathrm{~cm}^{-1} ;{ }^{1} \mathrm{H}$ NMR $\left(200 \mathrm{MHz}, \mathrm{CDCl}_{3}\right): \delta 8.25(1 \mathrm{H}, \mathrm{dd}$, $J=1.6,8.0 \mathrm{~Hz}, \mathrm{Ar}-\mathrm{H}), 7.65(1 \mathrm{H}, \mathrm{ddd}, J=1.7,7.3,8.5 \mathrm{~Hz}$, Ar-H), 7.29-7.18 (4H, m, Ar-H), 6.84 (1H, ddd, $J=0.9,2.6$, $8.2 \mathrm{~Hz}, \mathrm{Ar}-\mathrm{H}), 4.35\left(2 \mathrm{H}, \mathrm{ddd}, J=5.6,7.8,7.8 \mathrm{~Hz}, \mathrm{~N}-\mathrm{CH}_{2}\right)$, $4.20\left(2 \mathrm{H}, \mathrm{q}, J=7.1 \mathrm{~Hz}, \mathrm{CH}_{2}-\mathrm{CH}_{3}\right), 3.78\left(3 \mathrm{H}, \mathrm{s}, \mathrm{O}-\mathrm{CH}_{3}\right), 3.03$ $\left(2 \mathrm{H}, \mathrm{ddd}, J=5.6,8.4,8.4 \mathrm{~Hz}, \mathrm{Ar}-\mathrm{CH}_{2}\right), 1.30(3 \mathrm{H}, \mathrm{t}, J=7.2$ $\left.\mathrm{Hz},-\mathrm{CH}_{3}\right) \mathrm{ppm} ;{ }^{13} \mathrm{C} \mathrm{NMR}\left(50 \mathrm{MHz}, \mathrm{CDCl}_{3}\right): \delta 161.6,157.9$, $139.3,134.8,130.2,129.2,127.7,122.6,120.4,112.9,109.9$, 55.1, 41.6, 38.6, 29.6, 12.5 ppm. MS m/z: 325.1[M+H] $]^{+}$.

1-Ethyl-3-[2'-(3'-methoxyphenyl)ethyl]-1 H,3H-quinazoline-2,4-dione (4e,b). 1e $(0.10 \mathrm{~g}, 0.37 \mathrm{mmol})$ gave a white solid 4e,b (116.4 mg, 97\%); mp 112-114 ${ }^{\circ} \mathrm{C}$; (Lit. [16] mp. 112-114 ${ }^{\circ} \mathrm{C}$ ); IR (KBr): 3022, 2966, 2841, 1701, 1653, 1605 , 1483, $1258 \mathrm{~cm}^{-1} ;{ }^{1} \mathrm{H}$ NMR $\left(200 \mathrm{MHz}, \mathrm{CDCl}_{3}\right): \delta 8.25(1 \mathrm{H}$, $\mathrm{dd}, J=1.6,8.0 \mathrm{~Hz}, \mathrm{Ar}-\mathrm{H}), 7.68(1 \mathrm{H}, \mathrm{ddd}, J=1.7,7.3,8.5$ $\mathrm{Hz}, \mathrm{Ar}-\mathrm{H}), 7.34-7.20$ (4H, m, Ar-H), 6.91(1H, m, Ar-H), 6.77 $(1 \mathrm{H}, \mathrm{ddd}, J=0.9,2.6,8.2 \mathrm{~Hz}, \mathrm{Ar}-\mathrm{H}), 4.31(2 \mathrm{H}, \mathrm{ddd}, J=5.6$, 7.8, $\left.7.8 \mathrm{~Hz}, \mathrm{~N}-\mathrm{CH}_{2}\right), 4.20\left(2 \mathrm{H}, \mathrm{q}, J=7.1 \mathrm{~Hz}, \mathrm{CH}_{2}-\mathrm{CH}_{3}\right), 3.79$ $\left(3 \mathrm{H}, \mathrm{s}, \mathrm{O}-\mathrm{CH}_{3}\right), 2.97$ (2H, ddd, $\left.J=5.6,8.4 \mathrm{~Hz}, \mathrm{Ar}-\mathrm{CH}_{2}\right), 1.35$ $\left(3 \mathrm{H}, \mathrm{t}, J=7.2 \mathrm{~Hz},-\mathrm{CH}_{3}\right) \mathrm{ppm} ;{ }^{13} \mathrm{C} \mathrm{NMR}\left(50 \mathrm{MHz}, \mathrm{CDCl}_{3}\right): \delta$ $161.6,150.7,140.2,135.0,131.5,129.4,129.1,122.7,121.4$, $114.2,113.3,112.3,55.1,43.1,38.7,34.0,12.5$ ppm. ESI-MS $(\mathrm{m} / \mathrm{z}): 325.2[\mathrm{M}+\mathrm{H}]^{+}$.

1-Ethyl-3-[2'-(4'-methoxyphenyl)ethyl]-1H,3H-quinazoline-2,4-dione (4f,b). 1f $(0.10 \mathrm{~g}, 0.37 \mathrm{mmol})$ gave a white solid 4f,b (117.6 mg, 98\%); mp 110-112 ${ }^{\circ} \mathrm{C}$; (Lit. [16] mp. 110-112 $\left.{ }^{\circ} \mathrm{C}\right)$; IR (KBr): 3025, 2974, 2833, 1701, 1657, 1609, $1509,1483,1244 \mathrm{~cm}^{-1} ;{ }^{1} \mathrm{H} \mathrm{NMR}\left(200 \mathrm{MHz}, \mathrm{CDCl}_{3}\right): \delta 8.25$ $(1 \mathrm{H}, \mathrm{dd}, J=1.6,7.8 \mathrm{~Hz}, \mathrm{Ar}-\mathrm{H}), 7.66(1 \mathrm{H}, \mathrm{ddd}, J=1.7,7.3$, $8.4 \mathrm{~Hz}$, Ar-H), 7.29-7.18 (4H, m, Ar-H), 6.84(2H, ddd, $J=$ 1.6, 6.6, $6.6 \mathrm{~Hz}, \mathrm{Ar}-\mathrm{H}), 4.31(2 \mathrm{H}$, ddd, $J=5.6,7.8,7.8 \mathrm{~Hz}$, $\left.\mathrm{N}-\mathrm{CH}_{2}\right), 4.20\left(2 \mathrm{H}, \mathrm{q}, J=7.1 \mathrm{~Hz},-\mathrm{CH}_{2}-\mathrm{CH}_{3}\right), 3.77(3 \mathrm{H}, \mathrm{s}, \mathrm{O}-$ $\left.\mathrm{CH}_{3}\right), 2.97\left(2 \mathrm{H}, \mathrm{ddd}, J=5.6,8.2,8.2 \mathrm{~Hz}, \mathrm{Ar}-\mathrm{CH}_{2}\right), 1.35(3 \mathrm{H}, \mathrm{t}$, $\left.J=7.1 \mathrm{~Hz},-\mathrm{CH}_{3}\right) \mathrm{ppm} ;{ }^{13} \mathrm{C} \mathrm{NMR}\left(50 \mathrm{MHz}, \mathrm{CDCl}_{3}\right): \delta 161.6$, $156.2,158.2,150.3,139.5,135.0,130.7,129.9,129.1,122.7$, $115.9,113.7,113.3,55.2,43.3,38.7,33.0,12.5$ ppm. ESI-MS $(\mathrm{m} / \mathrm{z}): 325.1[\mathrm{M}+\mathrm{H}]^{+}$.

1-Ethyl-3-(2'-phenylethyl)-1 $\mathrm{H}, 3 \mathrm{H}$-quinazoline-2,4-dione (4g,b). 1 g (0.10 g, $0.41 \mathrm{mmol})$ gave a white solid 4g,b (117.0 mg, 97\%); mp 108-110 ${ }^{\circ} \mathrm{C}$; IR (KBr): 3033, 2929, 1705, 1657 , 1609, 1483, $1402 \mathrm{~cm}^{-1} ;{ }^{1} \mathrm{H}$ NMR $\left(200 \mathrm{MHz}, \mathrm{CDCl}_{3}\right): \delta 8.25$ $(1 \mathrm{H}, \mathrm{dd}, J=1.7,7.8 \mathrm{~Hz}, \mathrm{Ar}-\mathrm{H}), 7.68(1 \mathrm{H}, \mathrm{ddd}, J=1.7,7.3$, $8.4 \mathrm{~Hz}, \mathrm{Ar}-\mathrm{H}), 7.34-7.16(7 \mathrm{H}, \mathrm{m}, \mathrm{Ar}-\mathrm{H}), 4.29(2 \mathrm{H}, \mathrm{ddd}, J=$ 5.6, 7.8, $\left.7.8 \mathrm{~Hz}, \mathrm{~N}-\mathrm{CH}_{2}\right), 4.20\left(2 \mathrm{H}, \mathrm{q}, J=7.1 \mathrm{~Hz}, \mathrm{CH}_{2}-\mathrm{CH}_{3}\right)$, $2.98\left(2 \mathrm{H}, \mathrm{ddd}, J_{1}=5.2,8.0,8.0 \mathrm{~Hz}, \mathrm{Ar}-\mathrm{CH}_{2}\right), 1.34(3 \mathrm{H}, \mathrm{t}, J=$ $\left.7.1 \mathrm{~Hz},-\mathrm{CH}_{3}\right) \mathrm{ppm} ;{ }^{13} \mathrm{C} \mathrm{NMR}\left(50 \mathrm{MHz}, \mathrm{CDCl}_{3}\right): \delta 161.5$, $150.4,139.5,138.6,135.9,129.1,129.0128 .4,126.6,122.7$, $115.8,113.3,43.2,38.7,34.0,12.5$ ppm. ESI-MS $(m / z): 295.1$ $[\mathrm{M}+\mathrm{H}]^{+}$.

1-Ethyl-3-[3'-(4'-phenylpiperazin-1-yl)propyl]-1 H,3Hquinazoline-2,4-dione (4h,b). $1 \mathrm{~h}(0.10 \mathrm{~g}, 0.29 \mathrm{mmol})$ gave a light yellow solid 4h,b (109.2 mg, 96\%); mp 94-96 ${ }^{\circ} \mathrm{C}$; (Lit.
[16] mp. 94-96 $\left.{ }^{\circ} \mathrm{C}\right)$;IR (KBr): 3010, 2937, 2880, 2803, 1695, 1647, 1604, 1223, $1110 \mathrm{~cm}^{-1} .{ }^{1} \mathrm{H}$ NMR (200 MHz, $\left.\mathrm{CDCl}_{3}\right): \delta$ $8.10(1 \mathrm{H}, \mathrm{dd}, J=1.6,7.8 \mathrm{~Hz}, \operatorname{Ar}-\mathrm{H}), 7.73(1 \mathrm{H}, \mathrm{ddd}, J=1.8$, 7.4, $8.8 \mathrm{~Hz}$, Ar-H), $7.45(1 \mathrm{H}, \mathrm{d}, J=8.4 \mathrm{~Hz}, \mathrm{Ar}-\mathrm{H}), 7.31-7.14$ $(3 \mathrm{H}, \mathrm{m}, \mathrm{Ar}-\mathrm{H}), 6.89-6.72(3 \mathrm{H}, \mathrm{m}, \mathrm{Ar}-\mathrm{H}), 4.22-4.04$ (4H, m, $\left.(\mathrm{CO}) \mathrm{N}-\mathrm{CH}_{2},(\mathrm{CO})_{2} \mathrm{~N}-\mathrm{CH}_{2},\right), 3.01\left(4 \mathrm{H}, \mathrm{m}, \mathrm{ArN}-\left(\mathrm{CH}_{2}\right)_{2}\right), 2.56-$ $2.40\left(6 \mathrm{H}, \mathrm{m}, \mathrm{N}\left(\mathrm{CH}_{2}\right)_{2}\right), 1.86\left(2 \mathrm{H}, \mathrm{dt}, J=7.0,7.2 \mathrm{~Hz}, \mathrm{CH}_{2}\right)$, $1.25\left(3 \mathrm{H}, \mathrm{t}, J=7.1 \mathrm{~Hz}, \mathrm{CH}_{3}\right) \mathrm{ppm} ;{ }^{13} \mathrm{C} \mathrm{NMR}(50 \mathrm{MHz}, \mathrm{CD}-$ $\left.\mathrm{Cl}_{3}\right): \delta 164.9,154.7,153.7,143.1,138.8,132.5,131.8,126.2$, $122.6,119.1,118.9,117.7,59.4,56.5,52.1,51.4,43.3,28.0$, 16.0 ppm. ESI-MS $(\mathrm{m} / \mathrm{z}): 393.2[\mathrm{M}+\mathrm{H}]^{+}$.

\section{Acknowledgements}

We gratefully acknowledge support for this project by Consejo Nacional de Ciencia y Tecnología (CONACyT, GRANT No SEP-2004-CO1-47835) and Dirección General de Educación Superior Tecnológica (DGEST) for support this project. Leticia Guerrero thanks CONACyT for the graduate scholarship and Universidad Autónoma de Nayarit for their support.

\section{Supplementary Materials}

${ }^{1} \mathrm{H}$ NMR and ${ }^{13} \mathrm{C}$ NMR spectra for all reactions products are available.

\section{References}

1. (a) Hayao, S.; Havera, H. J.; Strycker, W. G.; Hong, E. J. Med. Chem. 1969, 12, 936. (b) Russel, R. K.; Press, J. B.; Rampulla, R. A.; McNally, J. J.; Falotico, R.; Keiser, J. A.; Bright, D. A.; Tobia, A. J. Med. Chem. 1988, 31, 1786. (c) Shiau, C. Y.; Chern, J. W.; Tien, J. H.; Liu, K. C. J. Heterocyclic Chem. 1989, 26, 595. (d) Nishikawa, Y.; Shindo, T.; Ishii, K.; Nakamura, H.; Kon, T.; Uno, H. J. Med. Chem. 1989, 32, 583.

2. (a) Hayao, S.; Havera, H. J.; Strycker, W. G.; Leipzig, T. J.; Kulp, R. A.; Hartzler, H. E. J. Med. Chem. 1965, 8, 807. (b) Hayao, S. US Patent 3, 274, 194, 1965. (c) Li, X.; Lee Y. R.; Kim, S. H. Bull. Korean Chem. Soc. 2011, 32, 3480.

3. (a) Leysen, J. E.; Niemegeers, C. J. E.; Neuten, J. M.; Laduron, P. M. Mol. Pharmacol. 1982, 21, 301. (b) Darchen, F.; Scherman, D.; Laduron, P. M.; Henry, J. P. Mol. Pharmacol. 1988, 33, 672.

4. Dreyer, D. L.; Brenner, R. C. Phytochemistry 1980, 19, 935.

5. (a) Ellis, G. P. Synthesis of Fused Heterocycles. In: Chemistry of Heterocyclic Compounds. Wiley Interscience, 1987. (b) Cortez, R.; Rivero, I. A.; Somanathan, R.; Aguirre, G.; Ramirez, F.; Hong, E. Synth. Commun. 1991, 21, 285. (c) Nikpour, F.; Paibast, T. Chem. Lett. 2005, 34, 1438. (d) Li, Z.; Huang, H.; Sun, H.; Jiang, H; Liu, H. J. Comb. Chem. 2008, 10, 484. (e) Li, J.; Chen, X.; Shi, D.; Ma, S.; Li, Q.; Zhang, Q.; Tang, J. Org. Lett. 2009, 11, 1193.

6. (a) Wright W. B.; Tomcufcik A. S.; Chan, P. S.; Marsico, J. W.; Press, J. B. J. Med. Chem. 1987, 30, 2277. (b) Takai, H.; Obase, H.; Karasawa, A.; Kubo, K.; Shuto, K.; Kasuya, Y.; Shigenobu, K. Chem. Pharm. Bull. 1986, 34, 1907. (c) Rivero, I. A.; Espinoza, K.; Somanathan, R. Molecules 2004, 9, 609-616.

7. Marques, C. A.; Selva, M.; Tundo, P.; Montanari, F. J. Org. Chem. 1993, 58, 5765 . 
8. Fu, Y.; Baba, T.; Ono, Y. J. Catal. 2001, 197, 91.

9. Selva, M. Synthesis 2003, 2872.

10. Guerrero, L.; Rivero, I. A. Arkivoc 2008, (xi), 295.

11. (a) Wasserscheid, P.; Keim, W. Angew. Chem. Int. Ed. 2000, 39, 3772. (b) Brown, R. A.; Pollet, P.; McKoon, E.; Eckert, C. A.; Liotta, C. L.; Jessop, P. G. J. Am. Chem. Soc. 2001, 123, 1254. (c) Sheldon, R. Chem. Comm. 2001, 2399. (d) Sima, T.; Guo, S.; Shi, F.; Deng, Y. Tetrahedron Lett. 2002, 43, 8145 (e) Mo, J.; Xu, L.; Xiao, J. J. Am. Chem. Soc. 2005, 127, 751.

12. (a) Sun, J.; Fujita, S.; Arai, M. J. Organomet. Chem. 2005, 690, 3490. (b) Ju, H. Y.; Manju, M. D.; Park, D. W.; Choe, Y.; Park, S. W. React. Kinet. Catal. Lett. 2007, 90, 3.

13. (a) Caddick, S. Tetrahedron, 1995, 51, 10403. (b) Lidström, P.; Tierney, J.; Wathey, B.; Westman, J. Tetrahedron 2001, 57, 9225. (c) Shieh, W.-C.; Lozanov, M.; Repič, O. Tetrahedron Lett. 2003, 44, 6943.

14. (a) Selva, M.; Trotta, F.; Tundo, P. J. Chem. Soc., Perkin Trans. 2, 1992, 519. (b) Selva, M.; Marques, C. A.; Tundo, P. J. Chem. Soc., Perkin Trans. 1, 1994, 1323. (c) Shieh, W.-C.; Dell, S.; Repič, O. J. Org. Chem. 2002, 67, 2188.

15. X-ray data has been deposited at the Cambridge Crystallographic Data Centre (Deposit number CCDC 779877). Crystal data and structure refinement of 1-ethyl-3-(3-methoxyphenethyl)quinazoli ne-2,4(1H,3H)-dione (4fb) are included in Supplementary Materials.

16. Rivero, I. A.; Guerrero, L.; Espinoza, K.; Meza, M. C.; Rodriguez, J. R. Molecules 2004, 14, 1860-1868. 